\title{
Software educativo en Lenguaje para la producción de textos narrativos en la Institución Educativa № 64975 , Pucallpa
}

\author{
Language educational software for narrative texts production in the \\ Educational Institution $N^{\circ}$ 64975, Pucallpa
}

Yris Yolanda Bedoya Campos'

Universidad Nacional Intercultural de la Amazonia

\section{RESUMEN}

Objetivos: Determinar en qué medida la creación y aplicación de software educativo en Lenguaje mejorará la producción de textos narrativos en cuento, fábula y leyenda en estudiantes del segundo grado de secundaria de la I. E. N ${ }^{\circ}$ 64975 Húsares del Perú de Pucallpa. Métodos: Investigación cuantitativa, diseño preexperimental con pre y postest, muestra no probabilística, es decir intencionada y constituida por 30 estudiantes. Los instrumentos de medición fueron las pruebas escritas, validadas y confiabilizadas estadísticamente con un coeficiente del 95,00 \% y el 0,957 , respectivamente. Resultados: De los 30 estudiantes evaluados en el pre-test obtienen una media aritmética de 4,50 y en el postest 22,00, una mejora de 17,50 puntos; en la mediana en el pre-test alcanzan 04, mientras que en el postest 24,00 (diferencia de 20 puntos); en la moda, en el pre-test se tiene 04 mientras que en el posttest se tiene también 24,00. Conclusiones: Se ha establecido con un nivel de significancia del $5 \%$ y la prueba t de Student igual a $-6,736$, que la creación y aplicación del software educativo en Lenguaje ha mejorado significativamente la producción de textos narrativos en cuento, fábula y leyenda en los estudiantes del segundo grado de secundaria de la I. E. N $N^{\circ} 64975$ Húsares del Perú, de Pucallpa, período escolar 2013.

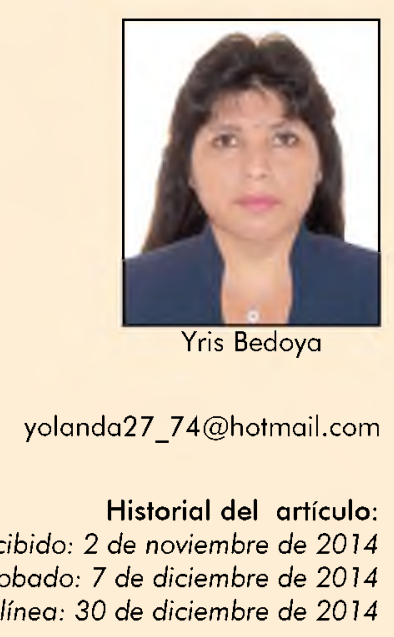

Palabras clave: Creación, aplicación, software educativo, lenguaje, producción de textos.

1 Lic. en Educación, especialidad Lengua y Literatura, Magister en Educación, abogada, docente asociada de la Universidad Nacional Intercultural de la Amazonia. 


\section{ABSTRACT}

Objectives: To determine how the implementation and application of Language educational software will improve the narrative texts production of tales, fairy tales and legends in second grade secondary students from Educational Institution $\mathrm{N}^{\circ} 64975$ Húsares del Perú, Pucallpa. Methods: Quantitative research, pre-experimental design with pre and posttest, nonrandom sample, so it's intentional and formed by 30 students. Measuring instruments were the written tests, validated and with statistical confidence in a coefficient of $95,00 \%$ and 0,957 respectively. Results: In the sample of 30 tested students, about the arithmetic mean, 4,50 in the pre-test and 22,00 in the post-test, an improvement of 17,50 points; about the median, 04 in the pre-test, while 24,00 in the post-test (difference of 20 points); about the mode, 04 in the pre-test while in the post test is 24,00 too. Conclusions: A significance level has been established with $5 \%$ and the Student's t test equal to $-6,736$, the implementation and application of language educational software has improved significantly the narrative texts production of tales, fairy tales and $d s$ in second grade secondary students from Educational Institution $N^{\circ} 64975$ Húsares del Perú, Pucallpa, 2013.

Keywords: Implementation, application, educational software, language, texts production, narrative texts, tales, fairy tales and legends.

\section{INTRODUCCIÓN}

Sobre el tema de la investigación son numerosos los trabajos realizados por distintos investigadores. A continuación mencionamos títulos de tesis realizadas a nivel internacional: Solevillas, Mori, Greco, Pautasso (1) Desarrollo de un soffware educativo para la enseñanza de la fotosíntesis, en la Universidad Nacional de Río Cuarto Facultad Cs. Exactas, Fco - Qcas y Naturales; asimismo Díaz, Rodríguez, Ruiz, Campos, Monteagudo, Alfonso y Cruz (2) El software educativo en la provincia de Sancti - Spíritus, en el Instituto Superior Pedagógico Capitán Silverio Blanco Núñez; asimismo Romero, Hernández y Henao (3) Diseño de un Software Educativo Multimedial y un aula virtual como apoyo a la asignatura historia de la pedagogía en Colombia para los programas de licenciatura de la facultad de educación en Uniminuto, en Colombia.

Asimismo tomamos como referencia a las investigaciones relacionados con nuestro tema a nivel nacional, así tenemos a Calsina (4) El docente ante las computadoras, en la Universidad Nacional Mayor de San Marcos; también citamos a Choque (5) Estudio en las aulas de Innovación Pedagógica y desarrollo de capacidades en tecnologías de información y comunicación - TIC en la Universidad Nacional Mayor de San Marcos; asimismo a Torres (6) Software Educativo que contribuye al desarrollo de habilidades en el cálculo aritmético en la escuela primaria Miguel Ruiz también en la Universidad Nacional Mayor de San Marcos; y finalmente tenemos a Mallqui y Palomino (7) Influencia del Software Educativo en el desarrollo de actitudes de aprendizaje en los alumnos del sexto grado de la I. E. N $N^{\circ} 32237$ Hipólito Unanue en la Universidad Nacional Hermilio Valdizán de Huánuco.

Respecto a las bases teóricas científicas, primero se denomina software educativo al que está destinado a la enseñanza y el aprendizaje autónomo y que, además, permite el desarrollo de ciertas habilidades cognitivas.

Así como existen diferencias entre las filosofías pedagógicas, también se encuentra una amplia gama de enfoques para la creación de soffware educativo, atendiendo a los diferentes tipos de interacción que se origina entre los actores del proceso de enseñanza-aprendizaje: educador, aprendiz, conocimiento, computadora. Existen principalmente dos tendencias: enfoque de instrucción asistida por computadora (Computer Assisted Instruction), y el enfoque de software educativo abierto. 
Vílchez (8) da una idea esencial de lo que es el texto narrativo al explicar cada componente del concepto. Sostiene que el texto es el conjunto coherente de enunciados que forma una unidad de sentido y que tiene intención comunicativa, mientras que el acto de narrar hace referencia a contar - referir una historia, tanto verídica como ficticia.

Por lo tanto puede decirse que el texto narrativo es aquel que incluye el relato de acontecimientos que se desarrollan en un lugar a lo largo de un determinado espacio temporal. Dicho relato incluye la participación de diversos personajes, que pueden ser reales o imaginarios.

Según Cóndor (9) "La narración está compuesta por una sucesión de hechos. En el caso de la narración literaria, inevitablemente configura un mundo de ficción, más allá de que los hechos narrados estén basados en la realidad". Esto sucede ya que el autor no puede abstraerse de incluir elementos de su propia invención o de matizar lo sucedido en el plano de lo real.

A nivel general, la estructura del texto narrativo está formada por una introducción (que permite plantear la situación inicial del texto), un nudo (donde surge el tema principal del texto) y un desenlace (el espacio donde se resuelve el conflicto del nudo).

Además de lo expuesto tendríamos que subrayar la existencia de dos tipos de estructuras. Por un lado, estaría la externa, que es la que se encarga de organizar la historia a través de capítulos, secuencias, etc. Por otro lado, la interna que es la que gira en torno al orden de los acontecimientos que van teniendo lugar.

Teniendo como referencia este sustento, se vislumbra que a lo largo de nuestra experiencia docente hemos dado cuenta que los estudiantes de Educación Básica Regular tienen pocas habilidades para la producción de textos narrativos en cuento, fábula y leyenda. Esta deficiencia también se observa en estudiantes que ingresan al nivel superior; debido a ello, se ha decidido investigar la creación y aplicación de Software Educativo en Lenguaje para la producción de textos narrativos en cuento, fábula y leyenda en los estudiantes del segundo grado de Secundaria de la I. E. $N^{\circ}$ 64975 Húsares del Perú de Pucallpa 2013.

Es importante que los estudiantes comprendan, en primer lugar, que cualquier conjunto de signos lingüísticos no necesariamente forman un texto. Solamente tendrá carácter de tal si presenta las siguientes características fundamentales: a) Coherencia, b) Cohesión, c) Concordancia, d) Ortografía.

Los estudiantes de la I. E. Húsares de Perú de Pucallpa, requieren escribir textos coherentes, es decir que las ideas guarden relación entre sí: palabras, párrafos y el texto en su totalidad a través de la presencia de un tema central y de subtemas específicos que se desprenden de aquel. También es necesario que utilicen la cohesión que establece las reglas para relacionar o articular palabras, frases y párrafos. Este principio busca que el texto no sea una suma de partes inconexas, sino una unidad en la que todos los elementos se relacionen entre sí. Los textos de los estudiantes deben establecer concordancia entre género, número y persona. Por último, los estudiantes del segundo grado de secundaria deben aprender las reglas ortográficas y aplicarlas en sus escritos, partiendo principalmente en la corrección de palabras que usan en su entorno y el quehacer diario.

Con el fin de mejorar esta dificultad, nuestro trabajo estuvo encaminado a investigar żen qué medida la creación y aplicación de Software Educativo en Lenguaje mejorará la producción de textos narrativos cuento, fábula y leyenda en los estudiantes del segundo grado de secundaria de la I. E. $N^{\circ}$ 64975 Húsares del Perú de Pucallpa?

El objetivo fue determinar en qué medida la creación y aplicación de un software educativo en Lenguaje mejorará la producción de textos narrativos en cuento, 
fábula y leyenda en los referidos estudiantes.

La hipótesis de investigación sostiene que la creación y aplicación del mencionado software educativo sí mejorará significativamente esa producción de textos narrativos en los alumnos.

\section{MATERIAL Y MÉTODOS}

Se ha utilizado el método experimental, con diseño pre experimental, cuyo esquema es el siguiente:

\section{G.E. : $O_{1} \quad X \quad O_{2}$}

Dónde:

G.E.: Grupo experimental

$\mathrm{X}$ : Experimento o variable independiente

$\mathrm{O}_{1}$ : Observación pre-test

$\mathrm{O}_{2}$ : Observación postest

La población estuvo constituida por todos los estudiantes del segundo grado de Educación Secundaria de la I. E. № 64975 Húsares del Perú de Pucallpa 2013; y la elección de la muestra de la investigación fue no probabilística por que no depende de la probabilidad sino de la preferencia en base a la experiencia del investigador, teniendo en cuenta, que los estudiantes poseen diferente estilo de aprendizaje, aún para demostrar que el uso de Software Educativo en lenguaje mejorará en los diferentes estilos de aprendizaje. Por ello se consideró a todos los estudiantes del 2 ㅇ grado de educación secundaria de la I. E. Nº4975 Húsares del Perú de Pucallpa que cuantitativamente fueron 30 estudiantes.

\section{RESULTADOS}

De la tabla $N^{\circ} 1$ y figuras $N^{\circ} 1$ y 2 , se puede deducir que de los 30 estudiantes evaluados del segundo grado, en el pre-test obtienen una media aritmética de 4,50 y en el posttest 22,00; es decir han mejorado 17,50 puntos; asimismo en la mediana en el pretest se tiene 04 mientras que en el post-test
Tabla $N^{0}$ 1: Resumen de estadísticos.

\begin{tabular}{|c|c|c|c|}
\hline & & Pre Test & Post Test \\
\hline \multirow[t]{2}{*}{$N$} & Valid & 11,00000 & 5,00000 \\
\hline & Missing & 19,00000 & 25,00000 \\
\hline \multicolumn{2}{|c|}{ Mean } & 4,63640 & 24,20000 \\
\hline \multicolumn{2}{|c|}{ Median } & 4,00000 & 24,00000 \\
\hline \multicolumn{2}{|c|}{ Mode } & 4,00000 & $19,000(a)$ \\
\hline \multicolumn{2}{|c|}{ Std. Deviation } & 2,46060 & 5,54076 \\
\hline \multicolumn{2}{|c|}{ Skewness } & $-0,07700$ & 1,14500 \\
\hline \multicolumn{2}{|c|}{ Std. Error of Skewness } & 0,66100 & 0,91300 \\
\hline \multicolumn{2}{|c|}{ Kurtosis } & $-0,92600$ & 1,33700 \\
\hline \multicolumn{2}{|c|}{ Std. Error of Kurtosis } & 1,27900 & 2,00000 \\
\hline \multicolumn{2}{|c|}{ Minimum } & 1,00000 & 19,00000 \\
\hline \multicolumn{2}{|c|}{ Maximum } & 8,00000 & 33,00000 \\
\hline \multicolumn{2}{|c|}{ Sum } & 51,00000 & 121,00000 \\
\hline
\end{tabular}

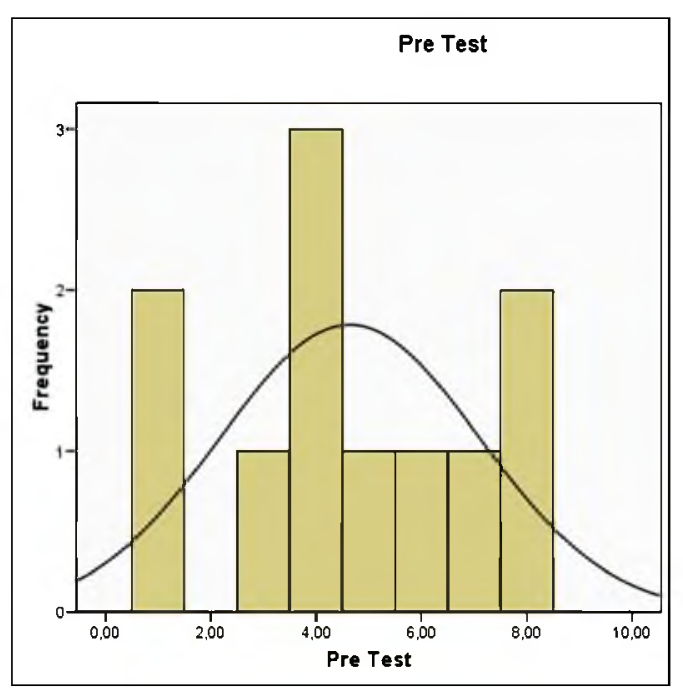

Figura $N^{0}$ 1: Resultados del pre-test.

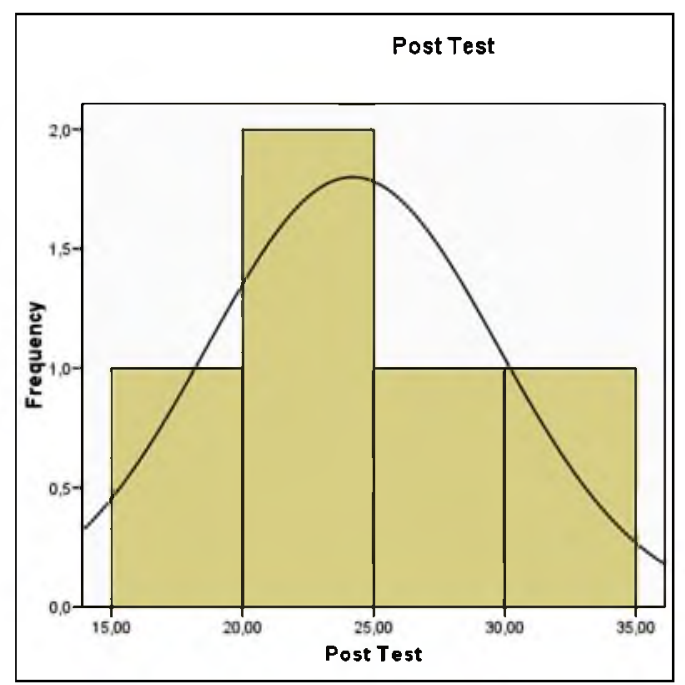

Figura No 2: Resultados del post-test. 
se tiene 24,00 (diferencia de 20 puntos); en la moda, en el pre-test se tiene 04 mientras que en el post-test se tiene también 24,00 (similar al caso anterior).

Como puntaje máximo se tiene el pre-test 08 , mientras que en el post-test 33 (diferencia de 25 puntos); respecto al puntaje mínimo en el pre-test se tiene 01 mientras que en el post-test se tiene 19 (diferencia de 18 puntos) siendo el rango en el primer caso 07 y en el segundo caso 14. En el pre-test se obtuvo una desviación estándar de 2,88 mientras que en el post-test se tuvo 2,94 deduciéndose que en el pre-test el grupo es ligeramente más homogéneo que en el post-test.

Siendo la $t$ calculada (tc) igual a: $-6,736$ y este cae en la zona de rechazo; entonces se rechaza la Ho, y acepta la hipótesis alterna $\mathrm{H} 1$.

Como se puede apreciar, se prueba la hipótesis estadística de investigación que a la letra dice: Si existe diferencias de medias entre el resultado del grupo experimental tanto en el pre-test como en el post-test. Y se ha demostrado que la creación y aplicación del software educativo en Lenguaje ha mejorado favorable y significativamente en la producción de textos narrativos cuento, fábula y leyenda en estudiantes del segundo grado de secundaria de la I. E. N 64975 Húsares del Perú de Pucallpa.

\section{DISCUSIÓN}

Como se ha demostrado con la t calculada (tc) igual a: $-6,736$ que se rechaza la $\mathrm{Ho}$, y se acepta la hipótesis alterna $\mathrm{H} 1$ que afirma que: La creación y aplicación del Software Educativo en lenguaje ha mejorado favorable y significativamente en la producción de textos narrativos cuento, fábula y leyenda en estudiantes del segundo grado de Secundaria de la I. E. N 64975 Húsares del Perú de Pucallpa.

Los resultados son corroborados con la investigación de Solevillas, Mori, Greco, Pautasso (1) donde concluye también que con el desarrollo de un soffware educativo se ha mejorado significativamente la enseñanza de la fotosíntesis.

También se ha demostrado que la creación y aplicación del software educativo en Lenguaje permite desarrollar capacidades comunicativas productivas en textos narrativos; asimismo constituye una herramienta pedagógica efectiva para la enseñanza-aprendizaje en producción de textos narrativos. Cabe mencionar que las experiencias logradas en el trabajo han permitido determinar la validez del programa Software Educativo ya que ha influenciado positiva y significativamente en el desarrollo de la producción de textos narrativos y en aprendizaje de los estudiantes de la muestra en estudio.

Asimismo, Romero, Hernández y Henao (3) también coinciden con nuestros resultados, ya que se ha determinado que merced a la aplicación del software educativo multimedial y el aula virtual, se ha mejorado favorablemente el rendimiento académico en la asignatura de historia de la pedagogía. Asimismo la investigación muestra una idea clara de la importancia del software educativo como herramienta de apoyo en el proceso de la enseñanza y el aprendizaje que puede ser utilizado en las distintas áreas y niveles educativos.

Para Torres (6) también refuerza nuestra investigación en la medida que el software contribuye al desarrollo de las habilidades en el cálculo aritmético. También el software contribuye como medio de enseñanza efectivo en el desarrollo del trabajo independiente de los alumnos, así como en las clases prácticas durante el proceso. Aquí el investigador nos muestra que el uso de software educativo en la matemática tuvo un resultado eficaz, demostrando de esta manera que a través de las imágenes y sonidos se aceleran los procesos mentales; de manera análoga en la producción de textos narrativos tuvo similar ocurrencia, siendo favorable para los estudiantes del segundo grado de Secundaria de la I.E N64975. Húsares del Perú de Pucallpa. 
En conclusión se ha determinado con un nivel de significancia del $5 \%$ que la creación y aplicación del software educativo en Lenguaje ha mejorado favorable $y$ significativamente en un $79,54 \%$ en la producción de textos narrativos cuento, fábula y leyenda en estudiantes del segundo grado de Secundaria de la I. E. N 64975 Húsares del Perú de Pucallpa.

La mejora mostrada por los referidos estudiantes en los textos narrativos, según las características textuales, es significativa en concordancia $(84,55 \%)$, coherencia $(74,52 \%)$ y cohesión $(54,56 \%)$, y es mediana en ortografía $(35,36 \%)$.

\section{REFERENCIAS BIBLIOGRÁFICAS}

1. Solivellas G, Morí L, Greco C, Romero D, Pautasso M, Joffre E. Desarrollo de un software educativo para la enseñanza de la fotosíntesis. [Tesis de pregrado]. Córdova: Universidad Nacional de Río Cuarto; 2005.

2. Díaz $A$, Rodríguez $M$, Ruiz $Y$, Campos $G$, Montenegro $O$, Alfonso $Y$, et al. El software educativo en la provincia de Sancti - Spíritus. [Tesis de pregrado]. Sancti Spiritus: Universidad de Ciencias Pedagógicas "Capitán Silverio Blanco Núñez"; 2000.

3. Romero $M$, Hernández J, Henao $P$ Diseño de un Software Educativo Multimedial y un aula virtual como apoyo a la asignatura historia de la pedagogía en Colombia para los programas de licenciatura de la facultad de educación en Uniminuto. [Tesis de pregrado]. Bogotá: Corporación Universitaria Minuto de Dios; 2008.

4. Calsina L. El docente ante las computadoras. $3^{\mathrm{a}}$ ed. Madrid: Gredos; 2005.

5. Choque R. Estudio en las aulas de Innovación Pedagógica y desarrollo de capacidades en tecnologías de información y comunicación - TIC. [Tesis de pregrado]. Lima: Universidad Nacional Mayor de San Marcos; 2009.
6. Torres JM. Software Educativo que contribuye al desarrollo de habilidades en el cálculo aritmético en la escuela primaria "Miguel Ruiz". [Tesis de maestría]. Sancti Spíritus: Centro Universitario "José Martí Pérez"; 2008.

7. Mallqui U, Palomino A, Santiago A. Influencia del Software Educativo en el desarrollo de actitudes de aprendizaje en los alumnos del sexto grado de la I.E. $N^{\circ} 32231$ Hipólito Unanue. [Tesis de pregrado]. Huánuco: Universidad Nacional Hermilio Valdizán; 2008.

8. Vílchez E. Gramática Básica. $4^{\underline{a}}$ ed. Lima: Pirámide; 2010.

9. Cóndor CA. Producción Literaria. 3ae ed. Lima: Pirámide; 2011. 